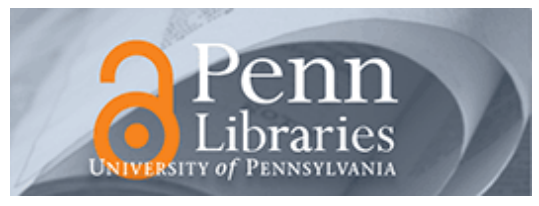

University of Pennsylvania

ScholarlyCommons

Marketing Papers

Wharton Faculty Research

6-2006

\title{
Procuring Fast Delivery: Sole Sourcing with Information Asymmetry
}

\author{
Gérard P. Cachon \\ University of Pennsylvania
}

Fuqiang Zhang

Follow this and additional works at: https://repository.upenn.edu/marketing_papers

Part of the Business Administration, Management, and Operations Commons, Business Analytics Commons, Business and Corporate Communications Commons, Management Sciences and Quantitative Methods Commons, Marketing Commons, and the Operations and Supply Chain Management Commons

\section{Recommended Citation}

Cachon, G. P., \& Zhang, F. (2006). Procuring Fast Delivery: Sole Sourcing with Information Asymmetry. Management Science, 52 (6), 881-896. http://dx.doi.org/10.1287/mnsc.1060.0510

This paper is posted at ScholarlyCommons. https://repository.upenn.edu/marketing_papers/285

For more information, please contact repository@pobox.upenn.edu. 


\title{
Procuring Fast Delivery: Sole Sourcing with Information Asymmetry
}

\begin{abstract}
This paper studies a queuing model in which a buyer sources a good or service from a single supplier chosen from a pool of suppliers. The buyer seeks to minimize the sum of her procurement and operating costs, the latter of which depends on the supplier's lead time. The selected supplier can regulate his lead time, but faster lead times are costly. Although the buyer selects the supplier to source from (possibly via an auction) and dictates the contractual terms, the buyer's bargaining power is limited by asymmetric information: The buyer only has an estimate of the suppliers' costs, while the suppliers know their costs precisely. We identify a procurement mechanism that minimizes the buyer's total cost (procurement plus operating). This mechanism is not simple: It is a numerically derived nonlinear menu of contracts.

Therefore, we study several simpler mechanisms: e.g., one that charges a late fee and one that specifies a fixed lead-time requirement (no menus, no nonlinear functions). We find that simple mechanisms are nearly optimal (generally within $1 \%$ of optimal) because asymmetric information conveys significant protection to the supplier, i.e., the supplier is able to retain most of the benefit of having a lower cost. Renegotiation is another concern with the optimal mechanism: Because it does not minimize the supply chain's cost, the firms can be both better off if they throw away the contract and start over. Interestingly, we find that the potential gain from renegotiation is relatively small with either the optimal or our simple mechanisms. We conclude that our simple mechanisms are quite attractive along all relevant dimensions: buyer's performance, supply chain performance, simplicity, and robustness to renegotiation.
\end{abstract}

\section{Keywords}

mechanism design, reverse auctions, supply chain coordination, game theory, renegotiation

\section{Disciplines}

Business | Business Administration, Management, and Operations | Business Analytics | Business and Corporate Communications | Management Sciences and Quantitative Methods | Marketing | Operations and Supply Chain Management 


\title{
Procuring Fast Delivery: Sole Sourcing with Information Asymmetry*
}

\author{
Gérard P. Cachon \\ Fuqiang Zhang \\ opim.wharton.upenn.edu/ cachon \\ opim.wharton.upenn.edu/ fuqiang \\ The Wharton School · University of Pennsylvania \\ Philadelphia PA, 19104
}

December 23, 2003, revised July 12, 2004

\begin{abstract}
This paper studies, in the context of a queuing model, a buyer that sources a good or service from an single supplier chosen from a pool of potential suppliers. The buyer seeks to minimize the sum of her procurement cost and her operating cost, the latter depends on the supplier's lead time performance. The selected supplier can regulate his lead time, but faster lead times are costly. Although the buyer selects the supplier to source from (possibly via an auction) and dictates the contractual terms, the buyer's bargaining power is limited by asymmetric information: the buyer only has an estimate of the suppliers' costs while the suppliers know their costs precisely. We identify procurement mechanisms for the buyer that minimize the buyer's total cost (procurement plus operating). These mechanisms are not simple: they involve a menu of contracts with non-linear functions that are derived numerically. Therefore, we study several simpler mechanisms, e.g., mechanisms that charge a late-fee and mechanisms that specify a fixed lead time requirement (no menus, no non-linear functions). We show that simple mechanisms can be nearly optimal: the buyer's cost is generally within $1 \%$ of optimal. Renegotiation is another concern with the optimal mechanisms: because they do not minimize the supply chain's cost, the firms can be both better off if they throw away the contract and start over. Interestingly, we find that the potential gain from renegotiation is relatively small with either the optimal or our simple mechanisms. Nevertheless, we also identify mechanisms that coordinate the supply chain (i.e., minimize its costs, thereby eliminating the threat of renegotiation).

${ }^{*}$ The authors would like to thank Martin Lariviere, William Lovejoy, Serguei Netessine, Erica Plambeck and Tunay Tunca for their helpful comments as well as the participants at the The Second MIT Symposium in Operations Research: Procurement and Pricing Strategies to Improve Supply Chain Performance. An electronic version of this paper is available from the authors' webpages. The previous version of this paper was titled "Procuring fast delivery, part II: sole sourcing with information asymmetry".
\end{abstract}


Those mechanisms are complex (due to asymmetric information) and they do not perform better for the buyer than our simple mechanisms. We conclude that our simple mechanisms are quite attractive along all relevant dimensions: buyer's performance, supply chain performance, simplicity and robustness to renegotiation.

Keywords: Mechanism design, reverse auctions, supply chain coordination, game theory, renegotiation 


\section{Introduction}

In the sourcing of a product or service, a buyer should consider both procurement price and delivery lead time. The faster a supplier's delivery lead time, the lower a buyer's operating costs (e.g., inventory holding and backorder penalty costs). A supplier's delivery lead time depends on the supplier's capacity, but capacity is costly, and so there is a classic incentive conflict within the supply chain: the supplier incurs the direct cost of capacity but the buyer enjoys its benefit. To complicate matters, the buyer often only has an estimate of the supplier's capacity cost, while the supplier knows it precisely.

While practitioners and academics surely understand the importance of lead times in the procurement process (see Burt, 1989; Pike and Johnson 2002; McNealy 2001; Wise and Morrison 2000), and the advent of the Internet has created an explosion of new marketplaces in the business-to-business arena (Pinker, Seidmann, Vakrat 2003), there has been surprisingly little research on how a buyer should design her procurement process to achieve minimum total cost through an effective balance of price and delivery lead time. That is the question this paper studies. Our main research questions are summarized as follows.

What is an optimal procurement mechanism for the buyer? A mechanism is any process that takes information the suppliers announce (e.g., their bid, their cost, etc.) and outputs the buyer's decisions: which supplier is chosen, what actions the suppliers must take and how much they are paid. The optimal mechanism minimizes the buyer's total cost (procurement plus operating) and it is the benchmark to assess all other mechanisms.

Do simple procurement mechanisms exist that give the buyer near optimal performance? The optimal mechanisms are complex along several dimensions: they may be hard to evaluate, or they may involve non-linear functions or a complex menu of functions. While we admit that there is no definitive way to measure how much "simpler" one mechanism is over another, this ambiguity should not cause research to focus exclusively on optimal mechanisms. We believe simple mechanisms are worth studying because they are more likely to be implemented in practice. Beil and Wein (2003) make a similar observation based on their discussions with industry practitioners.

To what extent is supply chain efficiency reduced by the buyer's desire to minimize her own total cost? The literature on supply chain coordination, which generally does not consider asymmetric information, suggests the buyer offer the supplier a coordinating contract (one 
that induces the supplier to choose the supply chain optimal capacity) and then negotiate for as large a share of the supply chain's profit as possible (e.g., Caldenty and Wein 2003). But implementing a coordinating contract is difficult with asymmetric information: the coordinating contract parameters may depend on the unknown information, thereby creating doubt with at least one firm as to what are the proper contract parameters. In addition, it is well known (see Laffont and Matrimort 2002) that ex post efficiency (i.e., maximizing supply chain performance) is at odds with the buyer's ex ante desire to maximize her own profit. This creates a renegotiation opportunity: after the optimal mechanism is implemented the firms have an incentive to scrap it to capture the lost efficiency. We wish to determine the magnitude of this trade-off in the context of our model.

The next section describes the model and $\S 3$ relates our work to the literature. $\S 4$ minimizes the supply chain's total cost. $\S 5$ covers procurement strategies with one potential supplier and $\S 6$ covers competitive bidding among multiple potential suppliers. $\S 7$ provides numerical results and $\S 8$ details two extensions to the model. The final section discusses our results.

\section{The model}

A buyer must acquire a component from one of $n \geq 1$ potential suppliers. The buyer uses this component in the assembly of a product sold to consumers. (In section 8.2 we assume the buyer is unable to hold component inventory, so in that case it is possible to interpret the model in terms of a buyer procuring a service rather than a physical product.) Customer demand arrives at the buyer according to a Poisson process with rate $\lambda$.

The suppliers are make-to-order manufacturers. Let $\mu$ be a supplier's production rate, which we generally refer to as the supplier's capacity. The supplier's inter-production times are exponentially distributed with mean $1 / \mu$ and the supplier incurs a capacity cost at rate $b \mu(b>0)$ to maintain its capacity. A potential supplier's capacity cost, $b$, is a realization of a random variable with finite support, $b \in[\underline{b}, \bar{b}]$ with $0<\underline{b} \leq \bar{b}$. Let $F$ and $f$ be the cdf and pdf respectively. We normalize the variable production cost to zero. Once the supplier completes the production of a unit it is immediately delivered to the buyer.

The buyer incurs inventory holding costs at rate $h$ per unit. A constant holding cost is reasonable if the physical holding cost plus the financial holding cost on the variable 
production cost dominates the financial holding cost due to the supplier's capacity cost and margin. Alternatively, a constant $h$ can be considered as an approximation for the holding cost given the possible range of procurement costs. Section 8 extends the model to allow the holding cost to vary with the procurement cost.

Unsatisfied demand is backlogged and the buyer incurs a goodwill cost at rate $p$ per backorder. The sum of the holding and backorder costs is referred to as the operating costs. To control her operating costs, the buyer uses a base-stock policy with base-stock level $s$.

The buyer's procurement strategy includes two tasks, supplier selection (which supplier to source from) and contract design (the details of the transfer payment between the buyer and the supplier). We consider several procurement strategies within two distinct scenarios. The first scenario is sole sourcing with one potential supplier $(n=1)$ : the buyer only offers a procurement contract to a single potential supplier, possibly because there is only one supplier with the necessary technology, or the buyer has a long-run relationship with the supplier, or because the buyer wishes to develop the component quickly. The next scenario involves competitive bidding among at least two potential suppliers $(n \geq 2)$, i.e., the buyer selects her supplier via some auction mechanism. (These are often called reverse auction because the suppliers are bidding for the right to sell to the buyer, but we shall just refer to them as auctions.) Within that scenario there are two versions: with identical suppliers the suppliers' have the same capacity cost whereas with heterogenous suppliers each suppliers' capacity cost is an independent draw from the same distribution. With either version the buyer knows the distribution from which the suppliers' capacity costs are drawn, but the buyer does not observe each supplier's cost realization. However, the buyer knows whether the suppliers are identical (have the same cost) or heterogenous (have different costs). All other rules and parameters in the game are common knowledge.

The sequence of events is as follows: the buyer announces her supplier selection process (some auction mechanism, if $n \geq 2$ ) and her transfer payment contract; assuming the supplier accepts the contract, the supplier chooses his capacity $\mu$; the buyer observes the supplier's lead times and chooses $s$; the buyer incurs costs (procurement and operating) and the supplier earns a profit (transfer payment minus capacity costs) over an infinite horizon. The buyer minimizes the sum of her procurement and operating costs per unit of time. The suppliers maximize their own expected profit per unit of time. All firms are risk neutral. 
Although we did not design this model with a specific industry in mind, the model is most representative of the contract manufacturing industry in which firms assemble specialized components on a make-to-order basis (see Thurm 1998; Bulkeley 2003).

\section{Literature review}

Our model studies procurement strategies in a queuing framework with asymmetric information. There is much related work, the closest of which is Cachon and Zhang (2003) (CZ). As in this paper, in CZ there is a single buyer with Poisson demand, the suppliers are make-to-order producers that choose capacity and the buyer is concerned with procurement and operating costs. However, this paper considers sole-sourcing strategies whereas CZ only works with multi-sourcing strategies. (For additional work on dual sourcing see Gilbert and Weng 1998, Ha, Li and Ng 2003, and Kalai, Kamien and Rubinovitch 1992.) Furthermore, this paper includes asymmetric information, whereas CZ does not.

Caldentey and Wein (2003) study a similar model to ours, but they do not consider supplier selection from a pool of potential suppliers and they do not have asymmetric information. They focus on coordination strategies whereas we consider the buyer's optimal mechanism. Benjaafar, Elahi and Donohue (2004) study multi-sourcing versus sole-sourcing strategies for a buyer that has several potential suppliers. They assume the buyer's price per unit is fixed and they do not include asymmetric information.

The following papers study a supply chain with two firms and asymmetric information in non-queuing models: Corbett and de Groote (2000), Corbett (2001), Corbett and Tang (1998), Corbett, Zhou and Tang (2001) and Ha (2001). As in this paper, those papers design an optimal menu of contracts, but we also consider a broader set of procurement strategies (e.g., competitive bidding and coordinating contracts). There is a literature on quality contracting with asymmetric information (e.g., Baiman, Fischer and Rajan 2000 and Lim 2001), but those models focus on the buyer's inspection decisions and the ability to contract on the outcome of inspections, neither of which is present in our model with lead times. There is work on supply chain signaling (e.g., Cachon and Lariviere 2001 and Ozer and Wei 2003). With a signaling model the party that possesses information also designs the contract whereas in our model the contract designer lacks information.

See Elmaghraby (2000) for a survey of the procurement literature, and see Klemperer 
(1999) and McAfee and McMillan (1987a) for surveys of the auction literature. Most closely related to our work is McAfee and McMillan (1987b), Laffont and Tirole (1987) and Che (1993). As in our paper, the first two articles study adverse selection (suppliers vary in their costs) with moral hazard (suppliers exert costly effort that benefits the buyer, where effort is analogous to capacity). Although there are some differences, we show that their results can be used to evaluate the optimal mechanism in our model. However, they do not study the effectiveness of simple mechanisms nor multiple suppliers with identical capacity costs. Che (1993) implements the optimal mechanism in McAfee and McMillan (1987b) and Laffont and Tirole (1987) via a scoring-rule auction in which the suppliers bid on both price and quality.

There are several other papers that study multi-attribute procurement. Chen, Roundy, Zhang and Janakiraman (2003) study procurement over price and transportation costs, but take the perspective of a third party auctioneer rather than the buyer. Manelli and Vincent (1995) consider (in effect) a multi-attribute situation in which the buyer's value is correlated with the suppliers' costs, i.e., the additional attribute is the supplier's identity. In our model the buyer is indifferent between any two suppliers as long as the suppliers have the same delivery time. Beil and Wein (2003) study multi-attribute auctions that occur over multiple rounds so that the buyer learns information regarding the suppliers in each round. We have a single round auction, so learning is not possible. They do not consider sole sourcing with only one potential supplier.

Dasgupta and Spulber (1990), Chen (2001), Hansen (1988), Jin and Wu 2002 and Seshadri and Zemel (2003) study procurement with competitive bidding and variable quantity. In our model the buyer's expected purchase quantity is fixed per unit time. There is a literature on lead time competition through operational strategies (e.g. Li 1992, Cachon and Harker 2002, So 2000), but in those papers the competitive structure is exogenous, whereas in our model it is endogenous. Ramasesh et al. (1991), Anupindi and Akella (1993), Sedarage et al. (1999) and Li and Kouvelis (1999) are representative studies investigating a buyer's procurement strategy given exogenous characteristics for each supplier (such as delivery time and price). There are a number of papers (see Cachon 1998, 2003 for surveys) that study supply-chain lead-time coordination in a multi-echelon inventory setting, but those papers do not have asymmetric information, nor do they consider procurement costs. We touch 
upon the issue of renegotiation. See Plambeck and Zenios (2000) and Plambeck and Taylor (2002) for other papers that discuss renegotiation, but in settings quite different than ours.

\section{Centralized management}

This section defines and derives several useful functions and presents the optimal policy for the supply chain. It is optimal for the supply chain to have one supplier (because the capacity cost is linear in $\mu$ ) and it is optimal to use a base-stock policy. Let $N$ be the number of outstanding orders at the supplier in steady state. $N$ is geometrically distributed. The buyer's operating cost is

$$
C_{o}(\mu, s)=E\left[h(s-N)^{+}+p(N-s)^{+}\right]=h\left(s-\frac{\lambda}{\mu-\lambda}\right)+(h+p)\left(\frac{\lambda}{\mu}\right)^{s}\left(\frac{\lambda}{\mu-\lambda}\right),
$$

the supplier's cost is $C_{s}(\mu, b)=b \mu$, and the supply chain's total cost is

$$
C(\mu, s, b)=C_{o}(\mu, s)+C_{s}(\mu, b)
$$

where $(x)^{+}=\max (0, x), \mu \geq 0$ and $s \in\{0,1,2, \ldots\}$. Because $s$ is restricted to the set of non-negative integers, it is not possible to provide a closed-form solution for the minimum cost. So in the remainder of this paper we treat $s$ as a continuous variable.

$C(\mu, s, b)$ is convex in $s$ and let $s^{*}(\mu)$ be the optimal base stock level:

$$
s^{*}(\mu)=-\ln \left(\left(\frac{h}{h+p}\right)\left(\frac{\mu / \lambda-1}{\ln (\mu / \lambda)}\right)\right) / \ln (\mu / \lambda) .
$$

Given the optimal base stock level, the buyer's operating cost is

$$
C_{o}(\mu)=C_{o}\left(\mu, s^{*}(\mu)\right)=h\left[\frac{1-\ln \left(\left(\frac{h}{h+p}\right)\left(\frac{\mu / \lambda-1}{\ln \mu / \lambda}\right)\right)}{\ln \mu / \lambda}-\frac{1}{\mu / \lambda-1}\right] .
$$

and the supply chain's total cost is

$$
C(\mu, b)=C\left(\mu, s^{*}(\mu), b\right)=C_{o}(\mu)+b \mu .
$$

According to the next theorem, $C_{o}(\mu)$ is convex in $\mu$. Consequently, $C(\mu, b)$ is convex in $\mu$. Let $\mu^{*}(b)$ be the supply chain's optimal capacity,

$$
\mu^{*}(b)=\arg \min _{\mu} C(\mu, b),
$$

and let $C^{*}(b)=C\left(\mu^{*}(b), b\right)$ be the supply chain minimum cost.

Theorem 1 The buyer's operating cost, $C_{o}(\mu)$, is convex in $\mu \geq \lambda$. 
All proofs are in the Appendix.

We later take advantage of an additional approximation of the supply chain's cost function. The exponential distribution is the continuous counterpart to the geometric distribution, so, as an approximation for $N$, we may use an exponential distribution with the same mean as the geometric distribution. This approximation tends to underestimate the average waiting time, but it is justified in a heavy traffic analysis (see Caldentey and Wein 2003). Let $\hat{C}(\mu, s, b)$ be the supply chain's cost function according to the exponential approximation:

$$
\hat{C}(\mu, s, b)=\hat{C}_{o}(\mu, s)+b \mu
$$

where $\hat{C}_{o}(\mu, s)$ is the buyer's operating cost,

$$
\hat{C}_{o}(\mu, s)=h s+\left(\frac{(h+p) e^{-s(\mu / \lambda-1)}-h}{\mu / \lambda-1}\right) .
$$

From Caldentey and Wein (2003), the unique global minimizers of $\hat{C}(\mu, s, b)$ are

$$
\hat{\mu}(b)=\lambda+\sqrt{\alpha / b} \text { and } \hat{s}(b)=\sqrt{b \alpha / h^{2}} \text {, where } \alpha=h \lambda \ln ((h+p) / h) .
$$

The buyer's minimum operating cost and the the supply chain's optimal cost are then

$$
\begin{aligned}
\hat{C}_{o}(\mu) & =\hat{C}_{o}(\mu, \hat{s}(b))=\alpha /(\mu-\lambda) \\
\hat{C}(b) & =\hat{C}(\hat{\mu}(b), \hat{s}(b), b)=b \lambda+2 \sqrt{\alpha b}
\end{aligned}
$$

We find that the supply chain's cost is nearly minimized with capacity $\hat{\mu}(b)$ as long as utilization is reasonably high (say more than 0.17). See Zhang (2004) for details.

\section{One potential supplier $(n=1)$}

In this section there is only one potential supplier (or the buyer has already selected her supplier), so the buyer only needs to set the transfer payment. We begin with the optimal mechanism, then consider supply chain coordination and finish with two simpler mechanisms.

\subsection{Buyer's optimal mechanism}

Although the space of possible contracts is quite large, according to the Revelation Principle, an optimal mechanism for the buyer is a menu of contracts that satisfies two constraints. The menu is a pair of functions, $\{\mu(x), R(x)\}$, such that the supplier chooses from this menu by announcing his cost to be $x$, then he builds capacity $\mu(x)$ and the buyer pays him $R(x)$

per unit produced. One constraint imposed on this menu is the incentive compatibility 
constraint:

$$
b=\arg \max _{x} \pi_{s}(x)=R(x) \lambda-b \mu(x),
$$

i.e., the supplier's true cost maximizes his profit, therefore he builds capacity $\mu(b)$ and receives $R(b)$ per unit delivered. The second is an individual rationality constraint:

$$
\pi_{s}(b) \geq 0 \quad \text { for all } b \in[\underline{b}, \bar{b}]
$$

i.e., the supplier participates only if his profit is non-negative (we assume zero profit is the supplier's best outside alternative). According to (3), the buyer designs a menu that even the highest cost supplier accepts, which implicitly assumes there is a severe penalty for failing to make an agreement with the supplier. (Corbett, Zhou and Tang 2001 relax this assumption in a different model.)

The buyer's total cost (procurement and operating) is $R \lambda+C_{o}(\mu)$, and the buyer's optimal menu is the solution to the following problem:

$$
\begin{aligned}
& \min _{\mu(\cdot), R(\cdot)} \int_{\underline{b}}^{\bar{b}}\left(R(x) \lambda+C_{o}(\mu(x))\right) f(x) d x \\
& \text { s.t. }(2),(3)
\end{aligned}
$$

Theorem 2 If $F(x)$ is log-concave, then the buyer's optimal menu of contracts to offer the supplier (i.e., the solution to (4)) is characterized by

$$
\begin{aligned}
C_{o}^{\prime}(\mu) & =-x-F(x) / f(x) \\
R(x) \lambda & =x \mu(x)+\int_{x}^{\bar{b}} \mu(y) d y .
\end{aligned}
$$

The log-concave requirement on $F(x)$ is sufficient (but not necessary) for the second order condition on each buyer's incentive compatibility constraint, (2). It is a mild restriction, satisfied by many commonly used distributions (see Bagnoli and Bergstrom 1989 for details.)

With the buyer's optimal mechanism the supplier builds less than the supply chain optimal capacity, $\mu^{*}(b)$ (the optimal capacity satisfies $C_{o}^{\prime}\left(\mu^{*}\right)=-b$, and $C_{o}(\mu)$ is convex), hence, the buyer sacrifices some ex post efficiency to increase her own profit. This is why the optimal mechanism is vulnerable to renegotiation: after the suppliers announces his capacity, both the buyer and the supplier can be better off if they renegotiate (choose $\mu^{*}(b)$ and a Pareto division of the supply chain's profit).

Because $C_{o}^{\prime}(\mu)$ is complex, we do not have a closed-form solution for $\mu(x)$ and $R(x)$. But it is possible to evaluate numerically the optimal menu and the buyer's expected cost. 


\subsection{Supply chain coordination (CC)}

Coordination requires that the supplier builds capacity $\mu^{*}(b)$, the supplier earns a nonnegative profit and the chosen base stock level is $s^{*}\left(\mu^{*}(b)\right)$. This can be done with the following arrangement: charge the supplier $h$ per unit in the buyer's inventory and $p$ per unit in the buyer's backorder, the supplier chooses $s$ and the unit price is

$$
R_{c}=C\left(\mu^{*}(\bar{b}), s^{*}(\bar{b}), \bar{b}\right) / \lambda .
$$

This works because the supplier incurs all supply chain costs, so the supplier has an incentive to choose $\mu^{*}(b)$ and $s^{*}\left(\mu^{*}(b)\right)$, and even the highest cost supplier earns a non-negative profit. The buyer's total cost is then $C\left(\mu^{*}(\bar{b}), s^{*}(\bar{b}), \bar{b}\right)$ and the supplier's profit is

$$
\pi_{s}=C\left(\mu^{*}(\bar{b}), s^{*}(\bar{b}), \bar{b}\right)-C\left(\mu^{*}(b), s^{*}(b), b\right) .
$$

This resembles Vendor Managed Inventory (because the supplier chooses $s$ ) with consignment and service penalties. Supply chain coordination is not achievable with a simpler mechanism: because only the supplier knows $b$, only a full transfer of the buyer's operating cost to the supplier results in the supplier choosing $\mu^{*}(b)$, and due to the full transfer of costs, the supplier must also choose the buyer's base stock level.

\subsection{Late-fee mechanism (LF)}

With a late-fee mechanism the buyer pays the supplier $R_{f}$ per unit and charges the supplier $\eta$ per outstanding order per unit time. This mechanism is simple to explain (just two parameters, no menu), easy to implement (it is based on data verifiable by both parties, the number of outstanding orders) and it is observed in practice (e.g., Beth et al. 2003). Although we would ideally like to find the optimal pair $\left\{R_{f}, \eta\right\}$, the complexity of $C_{o}(\mu, b)$ precludes a closed-form solution. As an alternative, we take advantage of the exponential approximation for $N$ to derive closed-form solutions for $R_{f}$ and $\eta$. We show in $\S 7$ that this approximation yields excellent results.

Let $C_{s}(\mu, b)$ and $\mu_{f}^{*}(b)$ be the supplier's cost and optimal capacity:

$$
\begin{aligned}
C_{s}(\mu, b) & =b \mu+\eta \lambda /(\mu-\lambda) \\
\mu_{f}^{*}(b) & =\arg \min _{\mu} C_{s}(\mu, b)=\lambda+\sqrt{\eta \lambda / b}
\end{aligned}
$$

Recall, $\hat{\mu}(b)=\lambda+\sqrt{\alpha / b}$ minimizes $\hat{C}(\mu, \hat{s}(b), b)$. Matching $\mu_{f}^{*}(b)$ with $\hat{\mu}(b)$ yields

$$
\eta_{f}=h \ln \left(\frac{h+p}{h}\right):
$$


if the late fee is $\eta_{f}$, then the supplier minimizes his cost with capacity $\hat{\mu}(b)$, which also happens to be the capacity that minimizes $\hat{C}(\mu, \hat{s}(b), b)$, the supply chain's cost function based on the exponential approximation. Hence, $\eta_{f}$ coordinates the approximate cost system. It does not coordinate the actual supply chain, but, as we already mentioned, it essentially does so when the optimal utilization is not too low.

To ensure participation, the buyer should pay $R_{f}$ per delivered unit such that

$$
\pi_{s}(\bar{b})=R_{f} \lambda-\bar{b} \hat{\mu}(\bar{b})-\eta_{f}\left(\frac{\lambda}{\hat{\mu}(\bar{b})-\lambda}\right)=0,
$$

which yields

$$
R_{f}=\bar{b}+2 \sqrt{\alpha \bar{b} / \lambda}
$$

Because $N$ does not depend on $s$, with the late-fee mechanism the buyer's optimal base stock level is $s^{*}(\hat{\mu}(b))$.

\subsection{Lead-time mechanism (LT)}

The lead-time mechanism is another simple mechanism for the buyer: the buyer merely tells the supplier the lead-time that must be delivered and how much the buyer pays for each unit. Due to the one-to-one relationship between the delivered lead time and the supplier's capacity, we can think of this mechanism in terms of two parameters, $\mu_{t}$ and $R_{t}$, the supplier's required capacity and the buyer's price per unit respectively. We assume the supplier must build capacity $\mu_{t}$ if the supplier accepts the contract, i.e., there is a substantial penalty for failing to adhere to the agreement. (We discuss this further in section 9.)

The supplier's expected profit is $\pi_{s}=\lambda R_{t}-b \mu_{t}$. To ensure participation, the unit price must be $R_{t}\left(\mu_{t}\right)=\bar{b} \mu_{t} / \lambda$. The buyer's cost is then

$$
C_{o}\left(\mu_{t}\right)+\lambda R_{t}\left(\mu_{t}\right)=C_{o}\left(\mu_{t}\right)+\bar{b} \mu_{t}
$$

which is the supply chain's cost with the highest capacity cost, $C\left(\mu_{t}, \bar{b}\right)$. Hence, the buyer's optimal lead time requirement is $\left(\mu_{t}-\lambda\right)^{-1}$, where $\mu_{t}=\mu^{*}(\bar{b})$, and the buyer pays the supplier $R_{t}\left(\mu^{*}(\bar{b})\right)$ per unit. Interestingly, from the buyer's perspective this mechanism is equivalent to the supply chain coordination mechanism. But unlike the supply chain coordination mechanism, the supply chain optimal capacity is chosen only when the supplier's cost is $\bar{b}$.

\section{Competitive bidding $(n \geq 2)$}

Now suppose there are at least two potential suppliers, so competitive bidding is possible. 
We evaluate an optimal mechanism and several other auction types when the suppliers' costs are identical or heterogeneous. With heterogeneous suppliers we employ the following random variables: let $\tilde{b}$ and $b_{l}$ be the lowest costs among $n-1$ and $n$ suppliers respectively, and let $b_{s}$ be the second lowest cost among $n$ suppliers. Let $G$ and $g$ be the cdf and pdf of $\tilde{b}$ and $b_{s}$ (i.e., they have the same distribution).

\subsection{A scoring-rule auction (SA)}

With a scoring-rule auction suppliers submit bids that contain a price and a lead time and the buyer evaluates these bids by assigning each bid a value via a publicly announced function (i.e., the scoring rule). Because from the buyer's perspective there is a one-to-one relationship between lead time and capacity, we shall assume, without loss of generality, that the suppliers submit $\{\mu, R\}$ bids, i.e., a capacity and a unit price. Let $Y(\mu, R)$ be the buyer's scoring rule and let $Y_{i}$ be the $i^{\text {th }}$ highest score. The winner is the supplier whose bid has the highest score. In the first-bid auction the winner must deliver his bid. With the second-bid auction the winner chooses any $\{\mu, R\}$ pair such that $Y_{2}=Y(\mu, R)$ (i.e., the winner does not have to exactly match the $2^{\text {nd }}$ best bid, he matches the $2^{\text {nd }}$ best bid's score.)

There are many possible scoring rules, but we work with an intuitive one: let the buyer's scoring rule be $Y_{B}(\mu, R)$, which is the buyer's total cost,

$$
Y_{B}(\mu, R)=C_{o}(\mu)+R \lambda
$$

So the highest score refers to the lowest total cost. Due to the next lemma, we can think of the suppliers as if they are bidding on $b \mu^{*}(b)+\pi_{s}$, the supply chain's optimal capacity cost plus a profit.

Lemma 3 In a (first or second bid) scoring-rule auction with the buyer's total cost as the scoring rule, $Y_{B}(\mu, R)$, the dominant strategy for a supplier with cost $b$ is to bid the supply chain optimal capacity $\mu^{*}(b)$.

Theorem 4 Consider the total cost, $Y_{B}(\mu, R)$, scoring-rule auction:

i. With identical suppliers: the suppliers bid $\left(\mu^{*}(b), R(b)\right)$ in equilibrium, where $R(x)=$ $x \mu^{*}(x) / \lambda$; the winner is randomly picked and earns zero profit; and this is an optimal procurement mechanism for the buyer (i.e., minimizes the buyer's total cost).

ii. With heterogeneous suppliers and first bid the unique symmetric equilibrium bidding 
strategy is

$$
\begin{aligned}
\mu(x) & =\mu^{*}(x), \\
R(x) \lambda & =-C_{o}\left(\mu^{*}(x)\right)+\frac{1}{(1-G(x))} \int_{x}^{\bar{b}} C^{*}(y) g(y) d y
\end{aligned}
$$

iii. With heterogeneous suppliers and second bid, it is a dominant strategy to bid according to $\mu(x)=\mu^{*}(x)$ and $R(x)=x \mu^{*}(x) / \lambda$.

With identical suppliers the buyer can coordinate the supply chain and extracts all its profit using a scoring-rule auction, even if she does not know the suppliers' cost. Therefore the total cost scoring-rule auction is optimal among all possible strategies. Furthermore, the buyer achieves this desirable outcome with as few as two suppliers.

With heterogeneous suppliers, Che (1993) proves the revenue equivalence result holds here, i.e., the buyer is indifferent between first bid and second-bid. (He considers bids on price and quality, but his results also apply here.) The intuition is straightforward: the suppliers are bidding on the buyer's total cost function, so the buyer only cares about her expected total cost, not the variance of her total cost. Che (1993) also shows that this scoring rule is not optimal for the buyer (the buyer is better off distorting the supplier to a lower than optimal capacity). This is also true in our model, as we next provide an optimal mechanism for heterogeneous suppliers.

\subsection{Optimal mechanism with heterogeneous suppliers (OM)}

Similar to the case of $n=1$, when $n \geq 2$ the buyer offers to the suppliers a menu, $\left\{q^{i}(\cdot), \mu^{i}(\cdot), R^{i}(\cdot)\right\}$, where $i \in[1, n]$ : supplier $i$ is the winner with probability $q^{i}(\hat{\mathbf{b}}) \geq 0$, where $\hat{\mathbf{b}}=\left(\hat{b}^{1}, \cdots, \hat{b}^{n}\right)$ is the vector of announced costs and $\sum q^{i}(\hat{\mathbf{b}})=1$; supplier $i$ receives a unit price $R^{i}(\hat{\mathbf{b}})$ from the buyer; the winner builds capacity $\mu^{i}(\hat{\mathbf{b}})$; and the losers do nothing but enjoy their payment.

Consider the suppliers' bidding behavior. Supplier $i$ maximizes her own expected profit:

$$
\max _{\hat{b}^{i}} \pi_{s}^{i}=E_{\hat{\mathbf{b}}^{-i}}\left[R^{i}(\hat{\mathbf{b}}) \lambda-q^{i}(\hat{\mathbf{b}}) b^{i} \mu^{i}(\hat{\mathbf{b}})\right] .
$$

According to the Revelation Principle, we need only consider truth telling mechanisms,

$$
b^{i}=\arg \max _{\hat{b}^{i}} \pi_{s}^{i}\left(\hat{b}^{i}\right) .
$$

The individual rationality constraints is

$$
\pi_{s}^{i}\left(b^{i}\right) \geq 0 .
$$


Let $\mathbf{b}=\left(b^{1}, \cdots, b^{n}\right)$ be the true cost vector. The buyer's problem is

$$
\begin{array}{ll}
\min _{\left\{q^{i}(\cdot), \mu^{i}(\cdot), R^{i}(\cdot)\right\}} & E_{\mathbf{b}}\left\{\sum_{i}^{n} R^{i}(\mathbf{b}) \lambda+\sum_{i}^{n}\left[q^{i}(\mathbf{b}) C_{o}\left(\mu^{i}(\mathbf{b})\right]\right\}\right. \\
\text { s.t. } & (7) \text { and }(8)
\end{array}
$$

The following theorem gives the solution to (9).

Theorem 5 If $F(\cdot)$ is log-concave then in the optimal mechanism for $n \geq 2$ heterogeneous suppliers the suppliers announce their true costs and the most efficient supplier is chosen. The same menu is offered to the suppliers with functions given by

$$
\begin{aligned}
q(\hat{\mathbf{b}}) & =\left\{\begin{array}{cc}
1 & \text { if } \hat{b}^{i}=\min \left(\hat{b}^{1}, \cdots, \hat{b}^{n}\right) \\
0 & \text { otherwise }
\end{array}\right. \\
C_{o}^{\prime}(\mu) & =-x-F(x) / f(x), \\
R(x) \lambda & =(1-F(x))^{n-1} x \mu(x)+\int_{x}^{\bar{b}}(1-F(y))^{n-1} \mu(y) d y,
\end{aligned}
$$

From Theorems (2) and (5) we see that the incentive scheme (i.e., the capacity function $\mu(x))$ applies for all $n$. So, again, the optimal mechanism results in less capacity than optimal for the supply chain. We numerically evaluate the functions in Theorem 5 .

This optimal mechanism is strange in that the losers receive a payment even though they do not build any capacity. However, it is possible to show that the optimal mechanism can be implemented so that only the winner receives a payment (see Zhang 2004).

\subsection{Lead-time mechanism with a price auction (LT)}

One idea to simplify the scoring-rule auction is to reduce its dimensionality: fix one of the dimensions and have the suppliers bid on the other dimension. In the lead time mechanism with a price auction the buyer announces the lead time the selected supplier must deliver and the selected supplier is the winner of a price auction. (This is the natural extension of the lead time mechanism with one potential supplier to $n \geq 2$ potential suppliers.) FreeMarkets runs auctions like this; see Rangan (1998). As before, we analyze this mechanism as if the buyer announces a required capacity, $\mu$, instead of a lead time.

Theorem 6 Consider the lead time mechanism with price auction.

i. With $n$ identical suppliers, the unique equilibrium (with first or second bid) is $R(x)=$ $\mu x / \lambda$, where the required lead time is $(\mu-\lambda)^{-1}$. The winner earns zero profit and the buyer's expected total cost, $\mu E[b]+C_{o}(\mu)$, is convex in $\mu$.

ii. With $n$ heterogeneous suppliers, the equilibrium bidding strategy with first bid is

$$
\varphi^{I}(x)=\frac{\mu}{\lambda} E(\tilde{b} \mid \tilde{b}>x)
$$


and with second bid the weakly dominant strategy is $\varphi^{I I}(x)=\mu x / \lambda$; first bid and second bid yield the same total cost to the buyer, $\mu E\left[b_{s}\right]+C_{o}(\mu)$, which is convex in $\mu$.

In the price auction, because the required capacity is given, the suppliers essentially bid on their profit. Hence, the revenue equivalence result follows immediately (part 2 of Theorem 6). Given the buyer's total cost is convex in $\mu$, a numerical search finds the optimal required capacity (i.e., lead time).

\subsection{Late-fee mechanism with a price auction (LF)}

In the late fee mechanism with a price auction the buyer charges the winner of the price auction the late fee $\eta_{f}$ per outstanding order per unit time. This is similar to the lead time mechanism in that the selection of the supplier is based only on the suppliers' price bids, but it is different in that now the winning supplier is free to choose his capacity/lead time to minimize his own costs. Because the winner's price bid does not influence his capacity choice, the winner chooses capacity $\hat{\mu}(b)$. As a result, with either first or second bid the suppliers effectively bid their capacity cost, $b \hat{\mu}(b)$, plus a profit. As with one potential supplier, $\eta_{f}$ is not the buyer's optimal late fee, but we show in $\S 7$ that it is quite good. The results for this mechanism are summarized in the following theorem.

Theorem 7 Consider the late fee mechanism with a price auction and the late fee $\eta_{f}$.

i. With $n$ identical suppliers, the unique equilibrium bid (with first or second bid) is $R(x)=\hat{C}(x) / \lambda$. The winner is chosen randomly and earns zero profit.

ii. With $n$ heterogeneous suppliers: the unique equilibrium bidding strategy with first bid is

$$
\begin{aligned}
\mu(x) & =\hat{\mu}(x) \\
R(x) & =\frac{1}{\lambda(1-G(x))} \int_{x}^{\bar{b}} \hat{C}(y) g(y) d y=\frac{1}{\lambda} E(\hat{C}(\tilde{b}) \mid \tilde{b}>x)
\end{aligned}
$$

and with second bid the dominant strategy is $\mu(x)=\hat{\mu}(x)$ and $R(x)=\hat{C}(x) / \lambda$; First bid and second bid yield the same expected total cost to the buyer.

\section{Numerical study}

This section reports on a numerical study of the procurement strategies analyzed in the previous two sections. We constructed 144 scenarios from all combinations of the following parameters: $h=1, \lambda \in\{0.1,1,10,100\}, p \in\{3,40,200\}, b$ is uniformly distributed on the interval $[\underline{b}, \bar{b}]$ where $\underline{b}=\theta-\delta$ and $\bar{b}=\theta+\delta, \theta \in\{0.5,5,50,200\}$ and $\delta \in\{0.05 \theta, 0.1 \theta, 0.2 \theta\}$. 
We take the scenarios with $\delta=0.05 \theta$ to represent reasonably small uncertainty with respect to the suppliers' cost (within $5 \%$ of forecast) and the scenarios with $\delta=0.20 \theta$ to represent high uncertainty (it is unlikely that qualified suppliers would have costs that range more than $20 \%$ from the buyer's forecast). We fix $h$ to a single value, because it is easy to show that the buyer's cost depends on the ratios $p / h$ and $b /(p / h)$, so it is sufficient to vary $p$ and $b$ and hold $h$ fixed. Because backorder penalty costs are generally higher than holding costs, we allow $p$ to range from a low value of three times $h$ to a high value of two hundred times $h$. Similarly, because of economies of scale in queuing systems, we range the demand rate for a low of 0.1 to a high of 100 . Capacity costs range from very low, $\theta=0.5$, which generally results in low utilizations, to very high, $\theta=200$, which generally results in high utilizations. Table 1 contains a list of the strategies we evaluate and the mnemonics we use to identify each one in the subsequent tables. With the competitive bidding strategies we assume there are two potential suppliers and we consider both identical and heterogeneous suppliers.

Table 2 provides data on the performances of each strategy relative to the optimal mechanism. With a single potential supplier, both the supply chain coordination mechanism (CC) and the late-fee mechanism (LF) are nearly optimal. In fact, LF even performs slightly better than CC at the 90th percentile because (we conjecture) it makes the supplier build less capacity than optimal, just like the optimal mechanism. We found that $\eta_{f}$ is nearly the optimal late fee, so there is little value to numerically search for the optimal late-fee mechanism. Figure 1 illustrates this result for a sample of the scenarios. It also illustrates that it is possible to increase costs substantially with a poorly chosen late fee.

In competitive bidding and identical suppliers, the scoring-rule auction (SA) is optimal for the buyer. The performance of the late-fee mechanism (LF) is very close to SA, again, even though $\eta_{f}$ is chosen via an approximation. The percentage cost increase relative to SA is less than $0.36 \%$ among $90 \%$ of the scenarios. The lead-time mechanism (LT) performs slightly better than LF. However, Zhang (2004) reports in a broader numerical study that LT performs worse than the LF when there is significant (and probably unrealistic) uncertainty in the supplier's cost.

With two heterogeneous suppliers, the scoring rule auction (SA) is no longer optimal, but it still generates total costs that are close to the optimal mechanism. Both the lead time (LT) and the late-fee mechanisms (LF) generate good results for the buyer. LF is 
least effective when both the capacity cost and the demand rate are very low (e.g., $\theta=0.5$, $\lambda=0.1$ ) because then the exponential approximation is not accurate due to very low system utilization.

Overall, we see from Table 2 that the late fee and lead-time mechanisms perform quite well. To test the robustness of these mechanisms, we constructed another set of 144 scenarios that are identical to the first with the one exception that in each scenario the capacity cost distribution is changed from a uniform distribution with mean $\theta$ and range $[\theta-\delta, \theta+\delta]$ to a normal distribution with mean $\theta$ and standard deviation $\delta / 4$. Table 3 summarizes those results. In short, both mechanisms continue to perform well when the uncertainty in the suppliers' cost is normally distributed.

We find that the buyer is much better off with two potential suppliers than only one potential supplier (when optimal mechanisms are used): on average the buyer's cost is $7.8 \%$ lower with two identical suppliers and $5.3 \%$ lower with two heterogenous suppliers relative to just one potential supplier.

Table 4 provides data on the incentive to renegotiate with the mechanisms that do not coordinate the supply chain. We see that the optimal mechanisms do create some opportunity for renegotiation, but that opportunity is generally relatively small (less than $1 \%$ for all scenarios). The late-fee mechanism also presents a small opportunity for renegotiation in most of the scenarios, except if the system utilization is very low (e.g. when the capacity cost and demand rate are very low). Again, this is because $\eta_{f}$ is derived from the exponential approximation, which is less accurate for systems with very low utilization. The renegotiation opportunity with the lead-time mechanisms is comparable to the late-fee mechanisms.

To summarize, we observe in an extensive numerical study that the lead time and latefee mechanisms perform for the buyer nearly as well as the optimal mechanisms and they generally create a relatively small renegotiation opportunity (i.e., they nearly coordinate the supply chain).

\section{Two extensions}

This section provides two extensions to the model: the buyer's holding cost is allowed to vary linearly with the buyer's procurement cost or the buyer operates in a make-to-order fashion, so the buyer does not hold inventory. 


\subsection{Generalized holding cost}

Now let $h$ be a function of the unit cost, $h=h_{0}+r c$, where $h_{0}$ is a constant representing the physical holding cost, $r$ is the interest rate and $c$ is the buyer's unit cost, which may differ from the unit price $R$. For example, the buyer's unit cost with a late fee is $R$ minus the late fee per unit. The buyer's operating cost is

$$
C_{o}(\mu, c)=\left\{\begin{array}{cc}
\left(h_{0}+r c\right)\left[\frac{1-\ln \left(\left(\frac{h_{0}+r c}{h_{0}+r c+p}\right)\left(\frac{\mu / \lambda-1}{\ln \mu / \lambda}\right)\right)}{\ln \mu / \lambda}-\frac{1}{\mu / \lambda-1}\right] & \text { if } s^{*}(\mu)>0 \\
\frac{p}{\mu / \lambda-1} & \text { if } s^{*}(\mu) \leq 0
\end{array}\right.
$$

Due to the continuous approximation of $s, s^{*}(\mu)<0$ and hence $C_{o}(\mu, c)<0$ is possible, so we need to adjust $C_{o}(\mu, c)$ for those outcomes. (With a fixed holding cost we did not encounter any situation in which $C_{o}(\mu, c)<0$, so that adjustment was not necessary in those scenarios.)

Unfortunately, the evaluation of the optimal mechanism with this new holding cost structure is quite difficult. There are several complications. First, $C_{o}(\mu, c)$ is not always jointly convex nor everywhere differentiable, which prevents finding solutions via first order conditions. Second, the transfer payment and the operating cost are no longer separable (i.e., the transfer payment can no longer be chosen arbitrarily for a given capacity and operating cost), which significantly complicates the evaluation of the optimal transfer payment and capacity function. As a result, full enumeration over the contract space is required to evaluate an optimal mechanism. Hence, we can only determine the optimal mechanism when the suppliers' costs are drawn from a discrete distribution and the suppliers are only allowed to choose capacities from a discrete set.

The process for evaluating the lead-time mechanism does not require an adjustment due to the variable holding cost. On the other hand, the late-fee mechanism requires an adjustment because the supply chain optimal capacity with the exponential approximation, $\hat{\mu}(b)$, no longer takes a simple form proportional to $\sqrt{1 / b}$. We first find the capacity that minimizes the supply chain's cost when $b=\theta$ (recall $\theta$ is the mean of the cost distribution):

$$
\mu_{\theta}=\arg \min _{\mu}\left(C_{o}(\mu, \theta)+\theta \mu\right)
$$

Unfortunately, there is no closed form solution for $\mu_{\theta}$, but only a one dimensional search is needed to find $\mu_{\theta}$. Given the late fee $\eta_{f}$, the supplier's optimal capacity is $\mu_{f}=\lambda+\sqrt{\eta_{f} \lambda / \theta}$. 
Equating $\mu_{\theta}$ with $\mu_{f}$ yields

$$
\eta_{f}=\theta\left(\mu_{\theta}-\lambda\right)^{2} / \lambda
$$

Hence, we set the late fee to coordinate the supply chain with the average cost supplier. With two or more suppliers, an auction sets the price $R$. With a single potential supplier, $R$ is chosen so that the high cost supplier earns zero profit:

$$
R_{f}=\bar{b}+2 \sqrt{\eta_{f} \bar{b} / \lambda}
$$

To test this version of the late-fee mechanism, we take the original set of 144 scenarios and add three interest rate levels, $r=\{0.05,0.1,0.2\}$, to arrive at 432 scenarios. For each scenario, we divide the cost support $[\theta-\delta, \theta+\delta]$ into $m-1$ equal intervals and assume each are equally likely (i.e., a discrete uniform distribution). Similarly, we divide the range $[1.1 \lambda, 10 \lambda]$ into $n-1$ equal intervals and use the $n$ interval boundaries as the feasible capacities. As $m$ and $n$ are increased, our discrete problem approaches the continuous problem we studied with a fixed holding cost. However, as we already mentioned, the computational burden increases rapidly with $m$ and $n$. In our numerical study, we set $m=5$ and $n=20$.

Table 5 displays the performance of the lead time and late-fee mechanisms relative to the optimal mechanisms. Even with this holding cost, the both mechanisms are nearly optimal with either a single supplier or identical suppliers. With heterogeneous suppliers the mechanisms perform well, but now the average cost increase is a noticeable $2.61 \%$ with either mechanism. We suspect that this gap with the optimal mechanism is in large part due to our coarse discretization because the gap decreases quickly as the number of supplier cost realizations $(m)$ increases. (For a sample of the scenarios we were able to test $m=7$ and $m=9$ ). From the supply chain's perspective, these mechanisms are also near optimal, so renegotiation is again not an issue (see Table 6). Overall, we conclude that the lead time and late-fee mechanisms perform quite well even when the holding cost is a linear function of the buyer's procurement cost.

\subsection{Make-to-order buyer}

If the buyer is a make-to-order manufacturer or a service provider, then the buyer is unable to hold buffer inventory to mitigate the consequence of slow delivery. Hence, we investigate whether the lead time and late-fee mechanisms perform well in this setting.

The buyer's operating cost is $C_{o}(\mu)=\lambda p /(\mu-\lambda)$. Theorem 5 still applies because $C_{o}(\mu)$ is convex, so we can evaluate the optimal mechanism for this case. It is easy to show 
that the buyer's optimal lead-time mechanism has $\mu_{t}=\lambda+\sqrt{p \lambda / \bar{b}}$ and the unit price $R_{t}\left(\mu_{t}\right)=\bar{b}+\sqrt{p \bar{b} / \lambda}$

There are two natural approaches for the late-fee mechanism. One approach is to transfer the buyer's backorder cost to the supplier: $\eta_{f}^{1}=p$ and $R=\bar{b}+2 \sqrt{p \bar{b} / \lambda}$. That approach coordinates the supply chain because the supplier incurs all operating costs and chooses all actions (in this case the only action is the capacity).

A second approach is to find the buyer's optimal late fee with one potential supplier. With the supplier's optimal capacity, $\mu_{f}^{*}(b)$, the supplier's profit is

$$
\pi_{s}\left(\mu_{f}, b\right)=R \lambda-\left(b \lambda+2 \sqrt{\eta_{f} \lambda b}\right)
$$

Setting $\pi_{s}\left(\mu_{f}, \bar{b}\right)=0$ gives the optimal transfer price:

$$
R_{f}=\bar{b}+2 \sqrt{\eta_{f} \bar{b} / \lambda}
$$

The buyer's expected cost with $\left\{\eta_{f}, R_{f}\right\}$ is

$$
C_{b}=\bar{b} \lambda+2 \sqrt{\eta_{f} \lambda \bar{b}}+\left(p-\eta_{f}\right) \sqrt{\lambda / \eta_{f}} E(\sqrt{b})
$$

which is convex in $\eta_{f}$ and minimized by

$$
\eta_{f}^{2}=\left(\frac{E(\sqrt{b})}{2 \sqrt{\bar{b}}-E(\sqrt{b})}\right) p .
$$

With heterogenous suppliers the unit price with either late-fee is determined via an auction.

Table 7 reports that all three of these procurement strategies perform well with the scenarios defined in $\S 7$. Comparing the two late-fee mechanisms, $\eta_{f}^{2}$ is better with one potential supplier (because then it is the optimal late fee) and two heterogeneous suppliers, and $\eta_{f}^{1}$ is better with identical suppliers (because then it is optimal). There is no renegotiation opportunity with $\eta_{f}^{1}$ because the supplier chooses the supply chain optimal capacity. The renegotiation values for the other strategies are given in Table 8: the supply chain inefficiency is positive but very small.

\section{Discussion}

A buyer procures a component from a single supplier whose capacity cost is unknown to the buyer. There are two tasks in the buyer's procurement strategy, supplier selection (which supplier to source from) and contract terms (how much to pay the supplier). Two 
situations are considered: with one potential supplier the buyer need only choose contract terms whereas with two or more potential suppliers the two procurement tasks (selection and contract terms) are bundled. With $n \geq 2$ we consider two versions: with identical suppliers the suppliers have the same capacity cost, whereas with heterogeneous suppliers each supplier's capacity cost is an independent draw from the same distribution.

In our base model the buyer holds inventory and the inventory holding cost is a constant. We identify optimal procurement strategies for the buyer and provide alternative strategies as well, in particular, simple mechanisms with a few fixed parameters rather than menus of non-linear functions. We judge each mechanism along two key dimensions: how well it minimizes the buyer's total cost (procurement plus operating) and how well it minimizes the supply chain's cost. If a mechanism does not score well on the latter dimension, then the mechanism may not be implementable due to the threat of renegotiation. Our main finding is that there exist simple mechanisms that are effective along both dimensions. One is a late-fee mechanism: the buyer charges the supplier fixed late fee for on-order units and either sets the unit price (with one potential supplier) or conducts an auction to set the unit price. The other is a lead-time mechanism: the buyer sets a fixed lead time requirement and uses the same procedure as the late-fee mechanism to set the unit price.

Although the lead time and late-fee mechanisms perform similarly, the lead-time mechanism has one disadvantage relative to the late-fee mechanism: compliance is harder to enforce. To explain, it is easy to imagine that the firms could disagree over whether the supplier has built the correct amount of capacity, because even if the correct capacity were built, the buyer cannot infer with certainty the supplier's capacity from the supplier's realized lead times. In other words, it is difficult for the buyer to distinguish between an unlucky (but honest) supplier and a cheating supplier. In contrast, it is easy for the firms to verify the number of outstanding orders, so a dispute is less likely to erupt between the parties with a late-fee mechanism.

For each procurement strategy with multiple potential suppliers we evaluated both a first bid and a second-bid auction. Even though these two auction formats have different equilibrium bidding strategies, we show that revenue equivalence holds in our setting, i.e., the buyer's expected total cost is the same with either format. However, this should not be taken to imply that revenue equivalence holds with all procurement strategies. Zhang 
(2004) demonstrates that revenue equivalence does not hold if the buyer sets a fixed price and conducts a lead time auction (i.e., suppliers bid a lead time and the winner is the one with the lowest lead time bid). With that mechanism the buyer prefers first bid over second bid because although the two have the same expected lead time bid, the variance of the lead time bids is lower with first bid. Interestingly, Zhang (2004) also shows that the buyer's cost with that mechanism, even with the first bid format, is significantly higher than optimal, which suggests that not all simple mechanisms are effective. Figure 1 further emphasizes this point: although the buyer's cost is relatively flat about the optimal late fee, it is possible to choose a late fee that significantly increases the buyer's cost.

To summarize, this research is about how a buyer should procure when both procurement and operating costs are important. It has been frequently articulated in the procurement literature that a buyer should not focus on just the purchase price, but rather on the total procurement cost. Unfortunately, there has been no rigorous analysis of how a buyer should go about balancing price with operating costs. The mechanism design literature suggests an approach that uses a menu of contracts to minimizes the buyer's total cost, albeit at the expense of supply chain inefficiency. The supply chain coordination literature seeks to maximize the supply chain's efficiency, but ignores the likely possibility of asymmetric information. Neither approach (mechanism design or supply chain coordination) values a simple design explicitly. Our practical approach is a blend of all three. For both simplicity and outstanding performance (the buyer's and the supply chain's), we recommend either the lead time or the late-fee mechanisms.

Table 1. Procurement strategies evaluated in the numerical study

OM Optimal mechanism (minimizes the buyer's cost)

CC Supply chain coordination mechanism (minimizes total supply chain cost)

LF Late-fee mechanism: the buyer pays $R_{f}$ per unit but charges the supplier the late fee $\eta_{f}$ per outstanding order per unit time. With one potential supplier, $R_{f}$ is chosen by the buyer, otherwise it is chosen via a price auction.

LT Lead-time mechanism: the buyer pays $R_{t}$ per unit and requires the supplier to achieve the lead time $\left(\mu_{t}-\lambda\right)^{-1}$. With one potential supplier, $R_{t}$ is chosen by the buyer, otherwise it is chosen via a price auction.

SA Scoring-rule auction: the scoring rule is $Y_{B}(\mu, R)$, which is the buyer's cost with unit price $R$ and capacity $\mu$; the winning supplier bids the lowest score and then must achieve his bid. 
Table 2. Cost comparisons among strategies. For each strategy, the percentiles of the percentage cost increase relative to the optimal strategy are presented.

\begin{tabular}{|c|c|c|c|c|c|c|c|c|c|}
\hline & & $\overline{\mathrm{min}}$ & $10 \%$ & $25 \%$ & $50 \%$ & $75 \%$ & $90 \%$ & $\max$ & average \\
\hline Single & $\mathrm{CC}$ and $\mathrm{LT}$ & $\overline{0}$ & 0.01 & 0.02 & 0.04 & 0.11 & 0.24 & 0.56 & 0.09 \\
\hline supplier & $\mathrm{LF}$ & 0.01 & 0.01 & 0.01 & 0.04 & 0.13 & 0.19 & 2.85 & 0.20 \\
\hline Identical & $\overline{\mathrm{LT}}$ & $\overline{0}$ & $\overline{0}$ & 0 & 0.01 & 0.04 & 0.10 & 0.24 & 0.03 \\
\hline suppliers & $\mathrm{LF}$ & 0 & 0 & 0 & 0 & 0.02 & 0.36 & 3.54 & 0.25 \\
\hline Heterogeneous & $\mathrm{SA}$ & 0.11 & 0.11 & 0.12 & 0.14 & 0.18 & 0.26 & 0.47 & 0.16 \\
\hline \multirow[t]{2}{*}{ suppliers } & LT & 0.11 & 0.11 & 0.12 & 0.14 & 0.19 & 0.31 & 0.59 & 0.18 \\
\hline & $\mathrm{LF}$ & 0.11 & 0.11 & 0.11 & 0.14 & 0.22 & 0.31 & 3.27 & 0.32 \\
\hline
\end{tabular}

Table 3. Performance of strategies when the capacity cost distribution is normal with mean $\theta$ and standard deviation $\delta / 4$. For each setting, the percentiles of the percentage cost increase relative to the optimal strategy are presented.

\begin{tabular}{crrrrrrrrr}
\hline \hline & & min & $10 \%$ & $25 \%$ & $50 \%$ & $75 \%$ & $90 \%$ & max & average \\
\hline Single & LT & 0.02 & 0.05 & 0.12 & 0.32 & 0.67 & 1.20 & 2.39 & 0.50 \\
supplier & LF & 0.01 & 0.05 & 0.11 & 0.34 & 0.79 & 1.38 & 3.53 & 0.60 \\
\hline Identical & LT & 0 & 0.01 & 0.01 & 0.01 & 0.01 & 0.02 & 0.12 & 0.01 \\
suppliers & LF & 0 & 0 & 0 & 0 & 0.02 & 0.35 & 3.54 & 0.25 \\
\hline Heterogeneous & LT & 0 & 0 & 0.01 & 0.02 & 0.03 & 0.06 & 0.12 & 0.02 \\
suppliers & LF & 0 & 0 & 0.01 & 0.01 & 0.03 & 0.27 & 3.40 & 0.23 \\
\hline
\end{tabular}

Table 4. The supply chain inefficiency (the value of renegotiation). The subscript $e$ denotes the expected supply chain inefficiency ex ante, the subscript max denotes the maximum possible supply chain inefficiency ex post.

\begin{tabular}{clrrrrrrrr}
\hline \hline & & $\min$ & $10 \%$ & $25 \%$ & $50 \%$ & $75 \%$ & $90 \%$ & max & average \\
\hline \multirow{5}{*}{ Single } & $\mathrm{OM}_{e}$ & 0 & 0 & 0.01 & 0.03 & 0.10 & 0.23 & 0.51 & 0.08 \\
supplier & $\mathrm{OM}_{\max }$ & 0 & 0.01 & 0.03 & 0.08 & 0.24 & 0.51 & 1.11 & 0.19 \\
& $\mathrm{LT}_{\text {max }}$ & 0 & 0.01 & 0.02 & 0.04 & 0.12 & 0.30 & 0.67 & 0.10 \\
& $\mathrm{LF}_{e}$ & 0 & 0 & 0 & 0 & 0.02 & 0.36 & 3.53 & 0.25 \\
& $\mathrm{LF}_{\max }$ & 0 & 0 & 0 & 0 & 0.02 & 0.45 & 4.38 & 0.27 \\
\hline \multirow{3}{*}{ Identical } & $\mathrm{LT}_{e}$ & 0 & 0 & 0 & 0.01 & 0.03 & 0.09 & 0.20 & 0.03 \\
suppliers & $\mathrm{LT}_{\max }$ & 0 & 0.01 & 0.01 & 0.04 & 0.11 & 0.34 & 0.73 & 0.11 \\
& $\mathrm{LF}_{e}$ & 0 & 0 & 0 & 0 & 0.02 & 0.36 & 3.53 & 0.25 \\
& $\mathrm{LF}_{\max }$ & 0 & 0 & 0 & 0 & 0.02 & 0.45 & 4.38 & 0.27 \\
\hline \multirow{5}{*}{ Heterogeneous } & $\mathrm{OM}_{e}$ & 0 & 0 & 0.01 & 0.02 & 0.06 & 0.14 & 0.30 & 0.05 \\
suppliers & $\mathrm{OM}_{\text {max }}$ & 0 & 0.01 & 0.03 & 0.08 & 0.24 & 0.51 & 1.11 & 0.19 \\
& $\mathrm{LT}_{\max }$ & 0 & 0 & 0.01 & 0.02 & 0.07 & 0.18 & 0.41 & 0.06 \\
& $\mathrm{LF}_{e}$ & 0 & 0.01 & 0.02 & 0.06 & 0.19 & 0.56 & 1.21 & 0.18 \\
& $\mathrm{LF}_{\max }$ & 0 & 0 & 0 & 0 & 0.02 & 0.39 & 3.78 & 0.25 \\
& & & 0 & 0 & 0.02 & 0.45 & 4.38 & 0.27 \\
\hline
\end{tabular}


Table 5. Performance of strategies with $h=h_{0}+r c$. For each setting, the percentiles of the percentage cost increase relative to the optimal strategy are presented.

\begin{tabular}{crrrrrrrrr}
\hline \hline & & $\min$ & $10 \%$ & $25 \%$ & $50 \%$ & $75 \%$ & $90 \%$ & max & average \\
\hline Single & LT & 0 & 0 & 0 & 0 & 0.10 & 0.66 & 2.22 & 0.15 \\
supplier & LF & 0 & 0 & 0 & 0 & 0.11 & 0.64 & 3.70 & 0.18 \\
\hline Identical & LT & 0 & 0 & 0 & 0 & 0 & 0.04 & 1.13 & 0.02 \\
suppliers & LF & 0 & 0 & 0 & 0 & 0 & 0.05 & 1.13 & 0.02 \\
\hline Heterogeneous & LT & 0.34 & 0.90 & 1.22 & 1.99 & 3.74 & 4.53 & 12.19 & 2.61 \\
suppliers & LF & 0.34 & 0.90 & 1.22 & 1.99 & 3.74 & 4.60 & 11.87 & 2.61 \\
\hline
\end{tabular}

Table 6. The supply chain inefficiency (the value of renegotiation) with $h=h_{0}+r c$. The subscript $e$ denotes the expected supply chain inefficiency ex ante, the subscript max denotes the maximum possible supply chain inefficiency ex post.

\begin{tabular}{clrrrrrrrr}
\hline \hline & & min & $10 \%$ & $25 \%$ & $50 \%$ & $75 \%$ & $90 \%$ & max & average \\
\hline \multirow{3}{*}{ Single } & $\mathrm{LT}_{e}$ & 0 & 0 & 0 & 0 & 0 & 0.06 & 1.43 & 0.04 \\
supplier & $\mathrm{LT}_{\max }$ & 0 & 0 & 0 & 0 & 0 & 0.23 & 4.89 & 0.14 \\
& $\mathrm{LF}_{e}$ & 0 & 0 & 0 & 0 & 0 & 0.06 & 1.13 & 0.03 \\
& $\mathrm{LF}_{\text {max }}$ & 0 & 0 & 0 & 0 & 0 & 0.21 & 4.89 & 0.09 \\
\hline \multirow{3}{*}{ Identical } & $\mathrm{LT}_{e}$ & 0 & 0 & 0 & 0 & 0 & 0.04 & 1.13 & 0.02 \\
suppliers & $\mathrm{LT}_{\max }$ & 0 & 0 & 0 & 0 & 0 & 0.17 & 4.89 & 0.09 \\
& $\mathrm{LF}_{e}$ & 0 & 0 & 0 & 0 & 0 & 0.06 & 1.13 & 0.03 \\
& $\mathrm{LF}_{\max }$ & 0 & 0 & 0 & 0 & 0 & 0.21 & 4.89 & 0.09 \\
\hline \multirow{3}{*}{ Heterogeneous } & $\mathrm{LT}_{e}$ & 0 & 0 & 0 & 0 & 0 & 0.04 & 2.21 & 0.02 \\
suppliers & $\mathrm{LT}_{\max }$ & 0 & 0 & 0 & 0 & 0 & 0.17 & 4.89 & 0.09 \\
& $\mathrm{LF}_{e}$ & 0 & 0 & 0 & 0 & 0 & 0.04 & 2.04 & 0.02 \\
& $\mathrm{LF}_{\text {max }}$ & 0 & 0 & 0 & 0 & 0 & 0.21 & 4.89 & 0.09 \\
\hline
\end{tabular}

Table 7. Performance of strategies with $s=0$. For each setting, the percentiles of the percentage cost increase relative to the optimal strategy are presented.

\begin{tabular}{clrrrrrrrr}
\hline \hline & & min & $10 \%$ & $25 \%$ & $50 \%$ & $75 \%$ & $90 \%$ & max & average \\
\hline & LT & 0 & 0.02 & 0.03 & 0.07 & 0.16 & 0.40 & 0.55 & 0.13 \\
Single & LF $\left(\eta_{f}^{1}\right)$ & 0 & 0.02 & 0.03 & 0.07 & 0.16 & 0.40 & 0.55 & 0.13 \\
supplier & LF $\left(\eta_{f}^{2}\right)$ & 0 & 0.01 & 0.01 & 0.02 & 0.04 & 0.08 & 0.12 & 0.03 \\
\hline \multirow{3}{*}{ Identical } & LT & 0 & 0 & 0.01 & 0.02 & 0.06 & 0.14 & 0.24 & 0.04 \\
suppliers & LF $\left(\eta_{f}^{1}\right)$ & 0 & 0 & 0 & 0 & 0 & 0 & 0 & 0 \\
\hline \multirow{3}{*}{ Heterogeneous } & LT & 0.11 & 0.01 & 0.02 & 0.05 & 0.13 & 0.33 & 0.39 & 0.10 \\
suppliers & LF $\left(\eta_{f}^{1}\right)$ & 0.11 & 0.11 & 0.13 & 0.17 & 0.25 & 0.43 & 0.51 & 0.21 \\
& & 0.10 & 0.11 & 0.11 & 0.13 & 0.16 & 0.20 & 0.24 & 0.14 \\
\hline
\end{tabular}


Table 8. The supply chain inefficiency (the value of renegotiation) with $s=0$. The subscript $e$ denotes the expected supply chain inefficiency ex ante, the subscript max denotes the maximum possible supply chain inefficiency ex post.

\begin{tabular}{clrrrrrrrr}
\hline \hline & & $\min$ & $10 \%$ & $25 \%$ & $50 \%$ & $75 \%$ & $90 \%$ & max & average \\
\hline \multirow{3}{*}{ Single } & $\mathrm{LT}_{e}$ & 0 & 0.01 & 0.03 & 0.08 & 0.19 & 0.49 & 0.58 & 0.15 \\
supplier & $\mathrm{LT}_{\text {max }}$ & 0 & 0.04 & 0.09 & 0.29 & 0.73 & 1.74 & 2.05 & 0.53 \\
& $\mathrm{LF}_{e}$ & 0 & 0.01 & 0.02 & 0.05 & 0.13 & 0.33 & 0.39 & 0.10 \\
& $\mathrm{LF}_{\max }$ & 0 & 0.01 & 0.02 & 0.06 & 0.14 & 0.33 & 0.39 & 0.11 \\
\hline \multirow{3}{*}{ Identical } & $\mathrm{LT}_{e}$ & 0 & 0 & 0.01 & 0.02 & 0.06 & 0.14 & 0.17 & 0.04 \\
suppliers & $\mathrm{LT}_{\max }$ & 0 & 0.01 & 0.02 & 0.08 & 0.22 & 0.53 & 0.62 & 0.16 \\
& $\mathrm{LF}_{e}$ & 0 & 0.01 & 0.02 & 0.05 & 0.13 & 0.33 & 0.39 & 0.10 \\
& $\mathrm{LF}_{\max }$ & 0 & 0.01 & 0.02 & 0.06 & 0.14 & 0.33 & 0.39 & 0.11 \\
\hline \multirow{3}{*}{ Heterogeneous } & $\mathrm{LT}_{e}$ & 0 & 0.01 & 0.01 & 0.05 & 0.12 & 0.29 & 0.35 & 0.09 \\
suppliers & $\mathrm{LT}_{\max }$ & 0 & 0.02 & 0.04 & 0.14 & 0.37 & 0.87 & 1.03 & 0.26 \\
& $\mathrm{LF}_{e}$ & 0 & 0.01 & 0.02 & 0.06 & 0.13 & 0.33 & 0.39 & 0.10 \\
& $\mathrm{LF}_{\max }$ & 0 & 0.01 & 0.02 & 0.06 & 0.14 & 0.33 & 0.39 & 0.11 \\
\hline
\end{tabular}

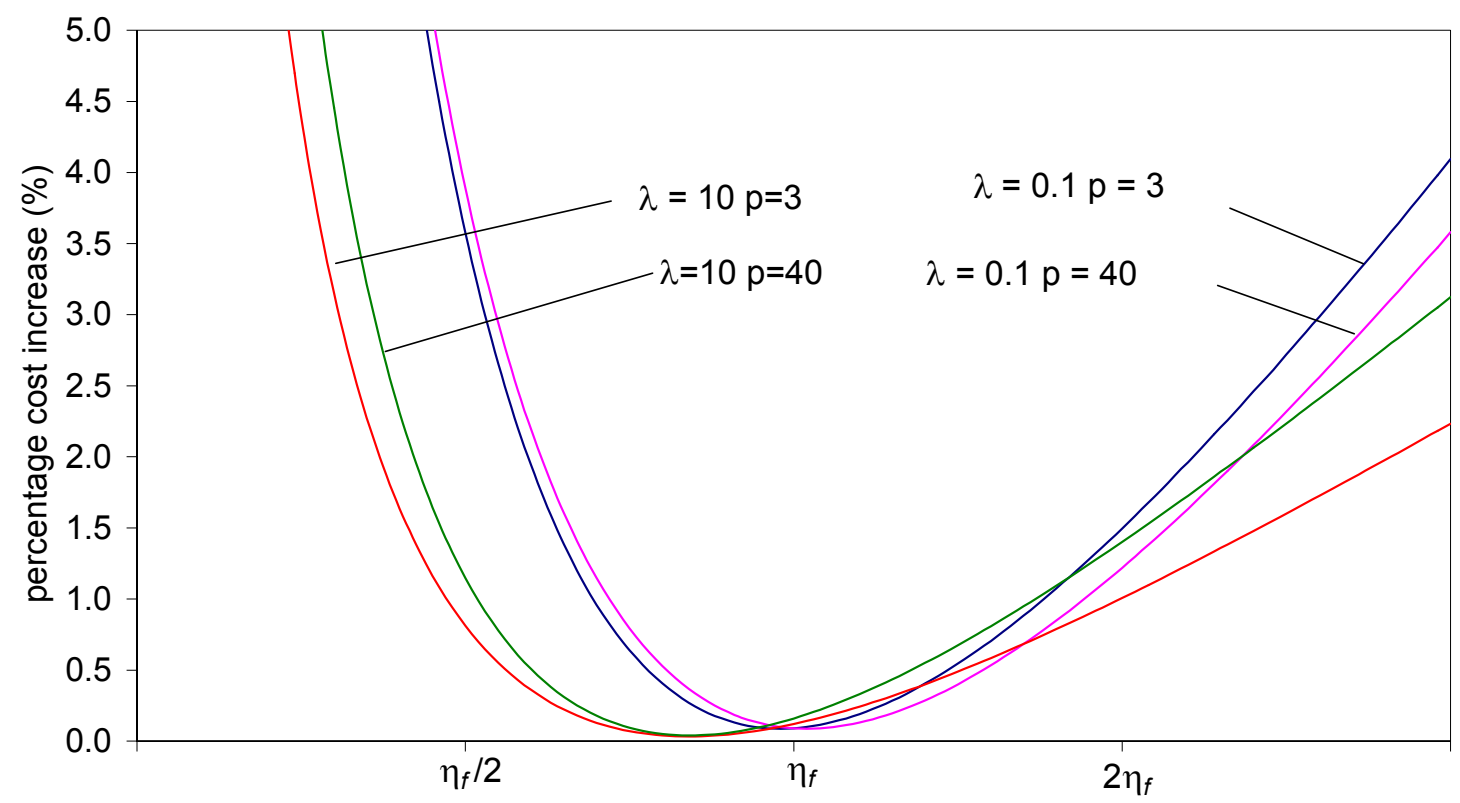

Figure 1: Buyer's total cost with a late-fee mechanism relative to the optimal mechanism for four scenarios with one potential supplier. The $\mathrm{x}$-axis is scaled so that in each scenario $\eta_{f} / 2, \eta_{f}$ and $2 \eta_{f}$ coincide. 


\section{Appendix}

Proof of Theorem 1: $C_{o}(\mu)$ is convex in $\mu$ if $C_{o}^{\prime \prime}(\phi) \geq 0$, where $\phi=\mu / \lambda$,

$$
\begin{aligned}
C_{o}^{\prime \prime}(\phi)= & h\left[\frac{-1-(\ln \phi+2)(\ln k+\ln (\phi-1)-\ln (\ln \phi))}{\phi^{2}(\ln \phi)^{3}}\right. \\
& \left.+\frac{2}{\phi(\phi-1)(\ln \phi)^{2}}+\frac{1}{(\phi-1)^{2} \ln \phi}-\frac{2}{(\phi-1)^{3}}\right],
\end{aligned}
$$

where $k=h /(h+p) . \quad C_{o}^{\prime \prime}(\phi) \geq 0$ if $g(\phi) \geq 0$ for $\phi>1$, where

$$
\begin{aligned}
g(\phi)= & 2 \phi(\phi-1)^{2} \ln \phi-(\phi-1)^{3}-2 \phi^{2}(\ln \phi)^{3}+\phi^{2}(\phi-1)(\ln \phi)^{2} \\
& -(\phi-1)^{3}(\ln \phi+2)(\ln (\phi-1)-\ln (\ln \phi)) .
\end{aligned}
$$

A simple plot reveals $g(\phi) \geq 0$ for all $\phi>1$. A more rigorous proof, based on Taylor series expansions, is provided by Zhang (2004)

Proof of Theorem 2: This is a special case of the proof for Theorem 5.

Proof of Lemma 3: For a fixed score y, the following program determines a supplier's bid, $(\mu, R)$, because the supplier's probability of winning depends only on $y$ :

$$
\begin{array}{ll}
\max _{\mu, R} & \pi_{s}=R \lambda-b \mu \\
\text { s.t. } & C_{o}(\mu)+R \lambda=y
\end{array} .
$$

Substitute the constraint into the profit function:

$$
\max _{\mu} \pi_{s}=y-C_{o}(\mu)-b \mu \Leftrightarrow \min _{\mu} C(\mu, b),
$$

i.e., the supplier chooses $\mu$ to minimize the system's total cost.

Proof of Theorem 4: From Lemma 3, $\mu^{*}(x)$ is a supplier's dominant strategy, so the supplier's profit is $R \lambda-b \mu^{*}(b)$. (i) given the suppliers know they have identical costs, the only equilibrium bidding strategy is $R(b)=b \mu^{*}(b) / \lambda$, which is the supplier's break even unit price: with any higher price there exists some other supplier that is willing to undercut the price by a small amount. (ii) We only need to consider the score, $y$, a supplier bids. Assume all suppliers implement the bidding strategy $\varphi(x)=C_{o}\left(\mu^{*}(x)\right)+R(x) \lambda$ and $\varphi(x)$ is decreasing. Supplier 1 wins if he submits the lowest score, i.e. if $x<\tilde{b}$ or equivalently $\varphi^{-1}(y)<\tilde{b}$. Supplier 1's expected profit is

$$
\begin{aligned}
\pi_{1} & =\left(y-C_{o}\left(\mu^{*}(x)\right)-x \mu^{*}(x)\right) \operatorname{Pr}\left(\tilde{b}>\varphi^{-1}(y)\right) \\
& =\left(y-C^{*}(x)\right)\left(1-G\left(\varphi^{-1}(y)\right)\right) .
\end{aligned}
$$

The first-order condition for the optimal $y$ is

$$
\left(1-G\left(\varphi^{-1}(y)\right)-\left(\varphi(x)-C^{*}(x)\right) \frac{g\left(\varphi^{-1}(y)\right)}{\varphi^{\prime}\left(\varphi^{-1}(y)\right)}=0 .\right.
$$


Because $x=\varphi^{-1}(y)$, rearranging the above yields,

$$
\frac{d}{d x}(\varphi(x)(1-G(x)))=C^{*}(x) g(x),
$$

which implies,

$$
\varphi(x)(1-G(x))=\int_{\underline{b}}^{x} C^{*}(y) g(y) d y+K,
$$

where $K$ is a constant. The left hand side is zero at $x=\bar{b}$. Equating the right hand side to zero at $x=\bar{b}$ yields

Combining (13) and (12) yields

$$
K=-\int_{\underline{b}}^{\bar{b}} C^{*}(y) g(y) d y
$$

$$
\varphi(x)=\frac{1}{1-G(x)} \int_{x}^{\bar{b}} C^{*}(y) g(y) d y=E\left(C^{*}(\tilde{b}) \mid \tilde{b}>x\right) .
$$

The result follows because $R(x) \lambda=\varphi(x)-C_{o}\left(\mu^{*}(x)\right)$. (iii) With second bid it is a dominant strategy to bid the minimum price the supplier is willing to receive, $R(x)=b \mu^{*}(x) / \lambda$.

Proof of Theorem 5: The proof is adapted from Laffont and Tirole (1987). We provide a sketch of the proof; Zhang (2004) provides a complete proof. A necessary condition for truth telling is

$$
\frac{\partial}{\partial \hat{b}^{i}} E_{\hat{\mathbf{b}}^{-i}} R^{i}(\hat{\mathbf{b}}) \lambda=\frac{\partial}{\partial \hat{b}^{i}} E_{\hat{\mathbf{b}}^{-i}}\left[q^{i}(\hat{\mathbf{b}}) b^{i} \mu^{i}(\hat{\mathbf{b}})\right] \text { at } \hat{b}^{i}=b^{i} \text { for all } i .
$$

We now assume that $q^{i}(\cdot)$ and $\mu^{i}(\cdot)$ are nonincreasing functions in $b^{i}$, and check later that they are indeed nonincreasing in the optimal mechanism. It follows that the first order condition (14) is sufficient for truth telling (see Zhang 2004).

Define $U^{i}\left(b^{i}\right)$ to be the expected profit for supplier $i$ under truth telling:

$$
U^{i}\left(b^{i}\right)=E_{\mathbf{b}^{-i}}\left[R^{i}(\mathbf{b}) \lambda-q^{i}(\mathbf{b}) b^{i} \mu^{i}(\mathbf{b})\right] .
$$

From (14) and (15) we have

$$
\dot{U}^{i}\left(b^{i}\right)=-E_{\mathbf{b}^{-i}}\left[q^{i}(\mathbf{b}) \mu^{i}(\mathbf{b})\right]
$$

We can see that $U^{i}$ is nonincreasing in $b^{i}$, so we can set

$$
U^{i}(\bar{b})=0, \text { all } i
$$

The buyer's problem now is

$$
\begin{array}{ll}
\min _{\left\{q^{i}(\cdot), \mu^{i}(\cdot), U^{i}(\cdot)\right\}} & E_{\mathbf{b}}\left\{\sum_{i}^{n} U^{i}(\mathbf{b})+\sum_{i}^{n}\left[q^{i}(\mathbf{b})\left(b^{i} \mu^{i}(\mathbf{b})+C_{o}\left(\mu^{i}(\mathbf{b})\right)\right]\right\}\right. \\
\text { s.t. } & (16) \text { and }(17)
\end{array}
$$


According to Zhang (2004), letting $\mu^{i}(\mathbf{b})$ be dependent on $b^{j}(j \neq i)$ is not optimal. So the above program can be simplified by only considering functions $\mu^{i}(\mathbf{b})$ that are functions of $b^{i}$ only. Once the optimal $q^{i}(\cdot)$ is given, so that $Q^{i}\left(b^{i}\right)=E_{b^{-i}} q^{i}(\mathbf{b})$ is given, the optimization with respect to $\mu^{i}\left(b^{i}\right)$ can be decomposed into $n$ programs as follows:

$$
\begin{aligned}
& \min \int_{\underline{b}}^{\bar{b}}\left\{U^{i}\left(b^{i}\right)+Q^{i}\left(b^{i}\right)\left[b^{i} \mu\left(b^{i}\right)+C_{o}\left(\mu\left(b^{i}\right)\right)\right] f\left(b^{i}\right) d b^{i}\right. \\
& \text { s.t. } \\
& \dot{U}^{i}\left(b^{i}\right)=-Q^{i}\left(b^{i}\right) \mu^{i}\left(b^{i}\right), \\
& U^{i}(\bar{b})=0 .
\end{aligned}
$$

This is a dynamic control problem with $U^{i}$ as the state variable and $\mu^{i}$ as the control variable. Solving this problem gives

$$
C_{o}^{\prime}\left(\mu^{i}\right)=-b^{i}-F\left(b^{i}\right) / f\left(b^{i}\right) .
$$

Since $\mu^{i}$ is the same for all $i$, we can drop the superscript. From (20), we have

$$
\int_{\underline{b}}^{\bar{b}} U^{i}\left(b^{i}\right) f\left(b^{i}\right) d b^{i}=\left.U^{i}\left(b^{i}\right) F\left(b^{i}\right)\right|_{\underline{b}} ^{\bar{b}}-\int_{\underline{b}}^{\bar{b}} F\left(b^{i}\right) d U^{i}\left(b^{i}\right)=\int_{\underline{b}}^{\bar{b}}\left[F\left(b^{i}\right) Q^{i}\left(b^{i}\right) \mu\left(b^{i}\right)\right] d b^{i} .
$$

Therefore, the cost function in (19) can be written as

$$
\int_{\underline{b}}^{\bar{b}} Q^{i}\left(b^{i}\right)\left[\frac{F\left(b^{i}\right)}{f\left(b^{i}\right)} \mu\left(b^{i}\right)+b^{i} \mu\left(b^{i}\right)+C_{o}\left(\mu\left(b^{i}\right)\right)\right] f\left(b^{i}\right) d b^{i}
$$

Let $A^{i}\left(b^{i}\right)=\frac{F\left(b^{i}\right)}{f\left(b^{i}\right)} \mu\left(b^{i}\right)+b^{i} \mu\left(b^{i}\right)+C_{o}\left(\mu\left(b^{i}\right)\right)$. From (22), we have

$$
\frac{d A^{i}}{d b^{i}}=\mu\left(b^{i}\right)\left(1+\frac{d}{d b^{i}} \frac{F\left(b^{i}\right)}{f\left(b^{i}\right)}\right)>0,
$$

so $A^{i}\left(b^{i}\right)$ is increasing in $b^{i}$. Hence we should give more weight to $Q^{i}\left(b^{i}\right)$ when $b^{i}$ is small. Since there are $n$ symmetric suppliers, the optimal $q^{i}(\cdot)$ must be $q^{i}(\mathbf{b})=1$ if $b^{i}<\min _{j \neq i} b_{j}$ and $q^{i}(\mathbf{b})=0$ otherwise. That is, in the optimal mechanism, the most efficient supplier is chosen with probability one. As a result, $Q^{i}\left(b^{i}\right)=\left(1-F\left(b^{i}\right)\right)^{n-1}$.

We can derive the profit function $U^{i}$ from (20) and (21):

$$
U^{i}\left(b^{i}\right)=\int_{b^{i}}^{\bar{b}}\left[(1-F(x))^{n-1} \mu(x)\right] d x .
$$

Again we can drop the superscript for $U^{i}$. The transfer payment function is therefore given by

$$
R\left(b^{i}\right) \lambda=\left(1-F\left(b^{i}\right)\right)^{n-1} b^{i} \mu\left(b^{i}\right)+\int_{b^{i}}^{\bar{b}}\left[(1-F(y))^{n-1} \mu(y)\right] d y
$$


Proof of Theorem 6: This proof follows the proof of Theorem 4. See Zhang (2004) for details.

Proof of Theorem 7: This proof follows the proof of Theorem 4. See Zhang (2004) for details.

\section{References}

Anupindi, R., R. Akella. 1993. Diversification under supply uncertainty. Mgmt Sci. 39(8) 944-963.

Bagnoli, M., T. Bergstrom. 1989. Log-concave probability and its applications. Working paper. University of Michigan.

Baiman, S., P. Fischer, M.V. Rajan. 2000. Information, contracting, quality costs. Mgmt Sci. 46(6) 776-789.

Beil, D., L. Wein. 2003. An inverse-optimization-based auction mechanism to support a multiattribute RFQ process. Mgmt Sci. 49(11). 1529-1545.

Benjaafar, S., E. Elahi, K. Donohue. 2004. Outsourcing via service quality competition. University of Minnesota working paper.

Beth, S., D. Burt, W. Copacino, C. Gopal, H. Lee, R. Lynch, S. Morris. 2003. Supply chain challenges: building relationships. Havard Busines Review. July.

Bulkeley, W. 2003. Plexus strategy: small runs of more things. Wall Street Journal. Oct 8.

Burt, D. 1989. Managing suppliers up to speed. Havard Business Review. July-Aug.

Cachon, G., 1998. Competitive supply chain inventory management. in Quantitative Models for Supply Chain Management. eds S. Tayur, R. Ganeshan, M. Magazine. Boston, Kluwer.

_. 2003. Supply chain coordination with contracts. The Handbook of Operations Research and Management Science: Supply Chain Management. eds S. Graves, T. de Kok, Kluwer.

_, P.T. Harker. 2002. Competition and outsourcing with scale economies. Mgmt Sci. 48(5) 1314-1333.

_, M. Lariviere. 2001. Contracting to assure supply: how to share demand forecasts in a supply chain. Mgmt Sci. 47(5) 629-646. 
_, F. Zhang. 2003. Obtaining fast service in a queueing system via performance-based allocation of demand. Working paper, University of Pennsylvania.

Caldentey, R., L.M. Wein. 2002. Analysis of a decentralized production-inventory system. MSOM. 5(1) 1-17.

Chayet, S., W. Hopp. 2002. Sequential entry with capacity, price, and leadtime competition. Working paper, Northwestern University.

Che, Y.-K. 1993. Design competition through multi-dimensional auctions. RAND Journal of Economics. 24(4). 668-680.

Chen, F. 2001. Auctioning supply contracts. Working paper, Columbia University.

Chen, R., R. Roundy, R. Zhang, G. Janakiraman. 2003. Efficient auction mechanisms for supply chain procurement. Cornell University working paper.

Corbett, C.J. 2001. Stochastic inventory systems in a supply chain with asymmetric information: cycle stocks, safety stocks, and consignment stock. Oper. Res. 49(4) 487-500.

Corbett, C.J., X. de Groote. 2000. A supplier's optimal quantity discount policy under asymmetric information. Mgmt Sci. 46(3) 444-450.

_, C.S. Tang. 1998. Designing supply contracts: contract type and information asymmetry. Chapter 9 in Tayur, S., R. Ganeshan, and M. Magazine (eds.), Quantitative Models for Supply Chain Management, Kluwer.

_, D. Zhou, C.S. Tang. 2002. Designing supply contracts: contract type and information asymmetry. Forthcoming in Mgmt Sci.

Dasgupta, S., D.F. Spulber. 1990. Managing procurement auctions. Information Economics and Policy. 4 5-29.

Elmaghraby, W.J. 2000. Supply contract competition and sourcing strategies. MSOM. 2(4) 350-371.

Gilbert, S.M., Z.K. Weng. 1998. Incentive effects favor nonconsolidating queues in a service system: the principle-agent perspective. Mgmt Sci. 44(12) 1662-1669.

Ha, A.Y. 2001. Supplier-buyer contracting: asymmetric cost information and cutoff level policy for buyer participation. Naval Research Logistics. 48 41-64.

Ha, A.Y., L. Li, S.-M. Ng. 2003. Price and delivery logistics competition in a supply chain. 
Mgmt Sci. 49(9) 1139-1153.

Hansen, R. 1988. Auctions with endogenous quantity. RAND J. of Econ. 19(1). 44-58.

Jin, M., S.D. Wu. 2002. Supply chain coordination in electronic markets: auction and contracting mechanisms. Working paper, Lehigh University.

Kalai, E., M. Kamien, M. Rubinovitch. 1992. Optimal service speeds in a competitive environment. Mgmt Sci. 38(8). 1154-1163.

Klemperer, P. 1999. Auction theory: a guide to the literature. Journal of Economics Surveys. 13(3) 227-286.

Laffont, J. J., D. Mattrimort. 2002. The Theory of Incentives: The Principal-Agent Model. Princeton, Princeton University Press.

Laffont, J.J., J. Tirole. 1987. Auctioning incentive contracts. Journal of Political Economy. 95(5) 921-937.

Li, C.-L., P. Kouvelis. 1999. Flexible and risk-sharing supply contracts under price uncertainty. Mgmt Sci. 45(10) 1378-1398.

Li, L. 1992. The role of inventory in delivery-time competition. Mgmt Sci. 38(2) 182-197.

Lim, W.S. 2001. Producer-supplier contracts with incomplete information. Mgmt Sci. 47(5) 709-725.

Manelli, A., D. Vincent. 1995. Optimal procurement mechanisms. Econometrica. 63 591-620.

McAfee, R.P., J. McMillan. 1987a. Auctions and bidding. Journal of Economics Literature. Vol. XXV 699-738.

McAfee, R.P., J. McMillan. 1987b. Competition for agency contracts. Rand Journal of Economics. 18(2) 296-307.

McNealy, S. 2001. Welcome to the bazaar. Havard Business Review. March. 2-3.

Özer, Ö., W. Wei. 2002. Strategic commitment for optimal capacity decision under asymmetric forecast information. Stanford University working paper.

Pinker, E., A. Seidmann, Y. Vakrat. 2003. Managing online auctions: current business and research issues. Mgmt Sci. 49(11). 1457-1484.

Plambeck, E., T. Taylor. 2002. Renegotiation of supply contracts. Stanford University working paper. 
_, S. Zenios. 2000. Performance-based incentives in a dynamic principal-agent model. MSOM. 2. $240-263$.

Pyke, D, E. Johnson. 2003. Sourcing strategies and supplier relationships: alliances vs. eprocurement. Forthcoming in The Practice of Supply Chain Management, eds. C. Billington, H. Lee, J. Neale, T. Harrison. Kluwer Publishing.

Rangan, V. K. 1998. FreeMarkets Online. Havard Business School case \#9-598-109.

Ramasesh, R.V., J.K. Ord, J.C. Hayya, A. Pan. 1991. Sole versus dual sourcing in stochastic lead-time (s, Q) inventory models. Mgmt Sci. 37(4) 428-443.

Sedarage, D., O. Fujiwara, H.T. Luong. 1999. Determining optimal order splitting and reorder level for N-supplier inventory systems. European Journal of Operational Research. 116 389-404.

Seshadri, S., E. Zemel. 2003. Supply intelligence. Working paper, NYU.

So, K.C. 2000. Price and time competition for service delivery. MSOM. 2(4) 392-409.

Thurm, S. 1998. Some U.S. manufacturers prosper by easing rise of 'virtual' firm. Wall Street Journal. Aug 18.

Wise, R., D. Morrison. 2000. Beyond the exchange: the future of B2B. Havard Business Review.(Nov-Dec). 86-96.

Zhang, F. 2004. Coordination of lead times in supply chains. Ph.D Dissertation. University of Pennsylvania. 